\title{
Zerrillo, Philip. (2019). The Case for Cases. Teaching with Cases. How to Teach Using the Case Method. Singapore: World Scientific. ISBN: 9789813273344. 94 pp.
}

\author{
Ana Almagro Esteban ${ }^{1}$ \\ https://orcid.org/0000-0002-7786-9252 \\ Universidad de Jaén \\ Facultad de Humanidades y Ciencias de la Educación, edificio D2. Paraje Las Lagunillas s/n 23071 Jaén.
}

Manual breve -aunque de vasta y sutil riqueza-, de lectura fácil y con un enfoque eminentemente práctico; al tiempo que radicalmente diferente, innovador en muchos casos y de suma utilidad. Este enfoque lo distingue de materiales clásicos en el campo, así como de las distintas novedades editoriales entre las que se encuentra. Su carácter diferenciador e innovador y su utilidad se sustentan en su practicidad que, a su vez, se fundamenta en una triple vertiente. De una parte, es un relato derivado de la experiencia de un experto en la aplicación del Método del Caso, que no persigue sino hacer llegar a un amplio espectro de profesores, recién iniciados y no tan noveles, la esencia de este método desde un punto de vista funcional. De otra, es un "collage" de consejos en distintas esferas, que esbozaremos a grandes rasgos. Y, finalmente, se centra en la preparación y aplicación del Método del Caso.

En los agradecimientos del manual ya se deja ver la sencillez de su autor, sencillez que suele ser sinónimo de conocimiento y experticia, además de generosidad. Esta es fácilmente constatable en cada una de sus líneas, que brindan un "goteo" incesante de consejos y apreciaciones exquisitas que difícilmente se descubren en la inagotable literatura en el campo.

Así, en el prólogo se delimita tanto el perfil del destinatario, esto es, "those that come to work each day and inspire the next generation of learners", como el principal cometido de este manual, encontrar nuestro propio estilo, el mejor para nuestros alumnos y el material más idóneo. Destaca también la introducción, que se estructura en 4 ejes:

(1) A partir de la pregunta de por qué utilizar el Método del Caso, se listan los atributos distintivos del mismo (realidad, relevancia, aprendizaje activo, conexión con las destrezas de identificación y resolución de problemas y la toma de decisiones y, por último, concienciación de los riesgos éticos de las decisiones). Entre todos estos atributos destaca su visión con respecto al último de ellos, la potenciación de la destreza comunicativa de las presentaciones -una de las grandes fortalezas de este método, aunque esta destreza había sido denostada durante mucho tiempo-. En esta línea, cabe destacar que la comunicación verbal y, por extensión, las presentaciones orales como exponente de la clara articulación de lo que comunicamos ocupan un lugar de privilegio desde la era Obama (Eaton, 2010, p. 13), aspecto en el que Zerrillo pone el dedo en la llaga.

(2) La apreciación personal del autor con respecto a este método y de la que como "un botón de muestra" sobresale tanto la correlación entre el Método del Caso y el consabido "can-do spirit" como la consecución de compartir y progresar, como evidencian sus palabras "the case method [...] helps our students to feel confident in sharing and stepping up" (p. 5).

(3) Una pregunta es de nuevo la vía para delimitar los riesgos de este método.

(4) Y, por último, la identificación, según este autor, de las tres Ps del Método, Plan, Prepare, Perform, que son la clave 
tanto para la inmersión exitosa de profesores y alumnos en el Método del Caso como para la consecución final de una carrera prometedora del alumno. Asimismo, es el momento oportuno de llamar la atención sobre un recurso recurrente de este manual a partir de cuadros de texto denominados "boxes", que destacan experiencias reales de otros expertos en el Método del Caso en distintas esferas.

Aparte de las secciones señaladas, la esencia del manual se concentra en 4 capítulos dedicados a la preparación del Método del Caso principalmente y, de forma menos extensa, a su aplicación. Así, la segunda de las dos Ps, Preparation, se despliega en la preparación en sí misma, la del alumno y la del profesor, correspondiéndose cada una de estas variables con los capítulos 2, 3 y 4 en cada caso.

El primero de ellos aborda cómo comenzar, siendo clave la esclarecedora apreciación de que cada profesor ha de identificar su propio estilo. Para ello resulta clave conocer a la audiencia (edad, género, experiencia, nivel de competencia en la lengua en la que se desarrolla la instrucción, los aspectos culturales y la actualidad de los temas propuestos), aspectos todos ellos que facilitan la elección de los casos prácticos. Esta elección es el punto neurálgico del capítulo, que se sustenta en diez criterios clave escrupulosamente desarrollados. Así, los mejores casos prácticos son aquellos que:

(1) Se corresponden con el currículum.

(2) Coinciden con los objetivos de aprendizaje.

(3) Son de naturaleza no multidimensional -“don't include the kitchen sink" (p. 20), como el propio autor señala-.

(4) Facilitan que el profesor sea el experto.

(5) Incluyen observaciones didácticas.

(6) Potencian que se escuche y se haga un diagnóstico del aula.

(7) Son fáciles de aplicar y se adaptan al estilo del profesor.

(8) Son adecuados para el nivel del alumno, aspecto que determinará la extensión, complejidad y la profundidad del caso práctico.

(9) Son interesantes y relevantes.

(10) Forman un todo con la institución, el plan de estudios y el grado -lo que se ejemplifica a partir de la expresión 'as "no man is an island""- (p. 28).

Así, se inicia el capítulo 3, dedicado a la preparación del alumno. Lo que sucede en el aula es equiparable, desde nuestro punto de vista, a la relación que se debe establecer entre las partes intervinientes en una negociación, esto es, "a win-win relation". Zerrillo, de otra parte, compara lo que acontece en el aula con una sinfonía que favorece algo esencial según sus palabras: "the deep peer-to-peer participation" (p. 34). Para ello, por una parte, el alumno debe conocer "las reglas del juego":

(1) Los beneficios de la preparación y las penalizaciones por la falta de esta.

(2) Lo que se considera participación aceptable y no aceptable.

(3) Los criterios de evaluación.

(4) Lo que no deben hacer en ningún caso.

(5) La correlación existente entre preparación y aprendizaje.

(6) El papel de la evaluación continua.

(7) Y, por último, las pautas de cómo prepararse.

Y, por otra parte, se recomienda proporcionar al alumno un modelo del género de escritura en cuestión cuando el caso práctico contemple una tarea escrita, sección en la que el autor ofrece orientación acerca de los errores típicos que se cometen en este tipo de actividad. Finalmente, la preparación del alumno se sustenta al mismo tiempo en cuestiones distintivas implícitas en cada caso práctico y cada día dedicado al mismo, como son: decidir las preguntas que ayudan al alumno a prepararse para sesiones de estas características, el material adicional, el papel que el alumno va a asumir en el caso práctico, si se ha de recabar información de distintos actores del caso práctico y, para concluir, si se va a indicar o no qué alumnos van a responder a qué cuestiones en concreto. 
La preparación del alumno se completa con la del profesor -que se describe como "rewarding and tough" (p. 51)-, contenidos que se introducen en el capítulo 4 y que son seminales en este manual. Así, inicialmente, hay una primera "parada" que equipa con reflexiones de primera necesidad: concebir la finalidad del caso práctico en cuestión y su conexión con los objetivos de aprendizaje, determinar su duración, delimitar la pregunta inicial, planificar cómo se van a utilizar las tecnologías y el papel de las mismas en el desarrollo del caso práctico, decidir cómo se va a concluir -el alumno espera conocer lo que debería haber aprendido y lo que ahora es capaz de hacer-, delimitar lo que ha aportado el caso práctico con respecto a los contenidos previamente introducidos y la progresión del currículum, clarificar la organización de los contenidos centrales del caso, determinar qué se va a revelar y qué no, cuantificar cuánto vamos a escuchar y a hablar y hacer uso de un archivo que recoja la evolución de nuestra visión del caso; reflexiones que, una vez introducidas, se desarrollan de forma detallada.

Y, así, de entre los contenidos de este capítulo, especial atención merecen cuestiones como el papel de la pregunta inicial del caso práctico -que se puede concebir como "recompensa" (favorecer que el alumno participe es la antesala de conseguir interesarlo), "castigo" o "barómetro" de la preparación del alumno-; la planificación del uso de la pizarra (la parte central para cuestiones clave, cálculos a ambos lados mejor que de arriba abajo, las conclusiones todas juntas a un lado u otro, entre otras muchas observaciones de suma relevancia); la metáfora del bosque y el claro en el bosque utilizada para ejemplificar las conclusiones que se deben esclarecer al alumno "it is a good idea to stop in the clearing and look back at the forest. Tell the students what they learned, how well they did on the path, and that now this part of the journey is complete, prepare them for the forest or hill that lies ahead" (p. 67); y lo que Zerrillo denomina las historias "post mortem", que funcionan como epílogo del caso y que son fruto de la documentación que se va archivando durante el desarrollo del mismo.

Llegados a este punto, la aplicación del Método del Caso y la tercera de las Ps, Performance, se abre hueco en el manual, contenidos que se corresponden con el capítulo 5. Según Zerrillo, cómo hacer uso de este método depende del estilo del profesor (taskmaster, cheerleader, conductor, facilitator, friendly mentor) (p. 89) y de aquello con lo que se siente cómodo, posición que suscribimos. Asimismo, se destaca que la forma en que se hace uso del Método del Caso depende, en gran medida, del tema del caso práctico, del número de alumnos y de su formación. No obstante, hay unas reglas de oro que se deben considerar: el profesor es el dueño de la clase - "Recognizing that you have the power to shape it is the first step in properly delivering" (p. 82)-es quien lleva la batuta, podríamos decir; no entrar en pánico -incluso si no se tiene una respuesta-; ser humano en el sentido de ser parte de lo que contamos; relacionar los casos prácticos con la vida de los alumnos; vigilar el ritmo de lo que hacemos; y, por último, ser flexibles. Al tiempo, estas reglas se completan con varios consejos de cómo hacer frente a ciertos problemas que pueden surgir en el aula (alumnos poco educados, los habladores, entre otras cuestiones)

Y, por último, el capítulo 6 -el más breve de todos ellos- introduce las conclusiones, cerrando el manual con un esquema de las variables que se deben considerar en la elaboración de un caso práctico. Así, ante las innumerables fortalezas de este manual, cabría considerar algunas cuestiones que contribuirían a subsanar un aspecto de carácter menor. Y nos referimos principal y exclusivamente a la nomenclatura de los epígrafes, que resulta difícil de seguir teniendo en cuenta el gran número de consejos que nos brinda, cuestión que, al mismo tiempo, evitaría la repetición de ciertos contenidos.

Centrándonos en trabajos previos en el campo, cabe subrayar que el manual que nos ocupa se distingue de todos ellos por su enfoque esencialmente práctico y resulta equiparable, en cierta medida, al extenso artículo de Golich (2000) "The ABCs of case teaching". Trabajo este de carácter académico que destaca por su exhaustividad, carácter integral y claridad. Recorre esta metodología desde las variables "The What" -¿qué es un caso, el Método 
del Caso, qué hace el profesor que hace uso de este método?-; "The Why" -¿por qué utilizarlo?; y "The How?" - ¿cómo preparase? Aquí se distinguen tres cuestiones claves: la esencia -hacer corresponder esta metodología con los resultados de aprendizaje y cómo seleccionar el caso práctico-, el proceso -distinción de las fases o "mapeo", las preguntas, escucha activa, validación de la participación del alumno y el uso de la pizarra- y el repaso o debriefing. Finalmente, estas variables se completan con un ejemplo de cómo preparar un caso práctico. Y, así, el manual de Zerrillo es la versión "en directo", esto es, la experiencia a pie de aula del trabajo de Golich. Esta experiencia se amplía y refrenda con la opinión en estilo directo de otros profesores de renombre que aplican el Método del Caso en distintas universidades internacionales.

Por todo ello, The Case for Cases. Teaching with Cases. How to Teach Using the Case Method abre una estela singular y enormemente enriquecedora que lo diferencia de otros manuales y que debería tomar una posición de privilegio como "mano derecha" en nuestra preparación para aplicar el Método del Caso.

\section{Declaration of conflicting interests}

The author(s) declared no potential conflicts of interest with respect to the research, authorship, and/or publication of this article.

\section{Funding}

The author(s) received no financial support for the research, authorship, and/or publication of this article.

\section{About the author}

Ana Almagro Esteban es Profesora Titular de la Universidad de Jaén, cuyas principales líneas de investigación y publicaciones se centran en Inglés para Fines Específicos, en las variedades de Inglés para los Negocios, Inglés para Turismo e Inglés para Arqueología y con especial atención al análisis de necesidades, el análisis y la evaluación de materiales didácticos y el Método del Caso.

\section{Referencias bibliográficas}

Eaton, S. E. 2010. Global Trends in Language Learning in the $21^{\text {st }}$ Century. Calgary: Onate Press.

Golich, V. L. 2000. “The ABCs of Case Teaching”. International Studies Perspectives, 1: 11-29.

Lima, M. y T. Fabiani. 2014. Teaching with Cases. A Framework Based Approach. London: Amazon.

Stanley, T. 2019. Case Studies and Case-Based Learning. Inquiry and Authentic Learning that Encourages 21st-Century Skills. Waco: Proofrock Press Inc.

Zerrillo, P. 2019. The Case for Cases. Teaching with Cases. How to Teach Using the Case Method. Singapore: World Scientific. 